\title{
Phase diagram and Chiral Magnetic Effect in Dirac Semimetals from Lattice Simulation
}

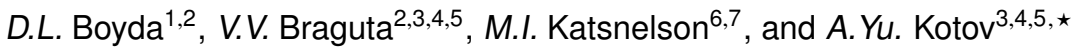 \\ ${ }^{1}$ Far Eastern Federal University, School of Natural Sciences, 690950 Vladivostok, Russia \\ ${ }^{2}$ Far Eastern Federal University, School of Biomedicine, 690950 Vladivostok, Russia \\ ${ }^{3}$ Institute of Theoretical and Experimental Physics, 117259 Moscow, Russia \\ ${ }^{4}$ Moscow Institute of Physics and Technology, Institutskii per. 9, Dolgoprudny, Moscow Region, 141700 \\ Russia \\ ${ }^{5}$ Joint Institute for Nuclear Research, BLTP, 141980 Dubna, Russia \\ ${ }^{6}$ Radboud University, Institute for Molecules and Materials, Heyendaalseweg 135, NL-6525AJ Nijmegen, \\ The Netherlands \\ ${ }^{7}$ Ural Federal University, Theoretical Physics and Applied Mathematics Department, Mira Str. 19, 620002 \\ Ekaterinburg, Russia
}

\begin{abstract}
Dirac Semimetals $\mathrm{Na}_{3} \mathrm{Bi}$ and $\mathrm{Cd}_{3} \mathrm{As}_{2}$ are recently discovered materials, which low energy electronic spectrum is described by two flavours of massless $3+1 \mathrm{D}$ fermions. In order to study electronic properties of these materials we formulated lattice field theory with rooted staggered fermions on anisotropic lattice. It is shown that in the limit of zero temporal lattice spacing this theory reproduces effective theory of Dirac semimetals. Using the lattice field theory we study the phase diagram of Dirac semimetals in the plane effective coupling constant - Fermi velocity anisotropy. We also measure conductivity of Dirac Semimetals within lattice field theory in external magnetic field. Our results confirm the existence of Chiral Magnetic Effect in Dirac Semimetals.
\end{abstract}

\section{Introduction}

Recent years in condensed matter physics are marked by discovery of new materials with unusual properties. Considerable interest attract materials, which low energy fermionic spectrum can be described by massless fermions. Probably one of the most famous examples is graphene[1, 2] - two-dimensional list of carbon atoms. Its three-dimensional analogues include $\operatorname{Dirac}\left(\mathrm{Na}_{3} \mathrm{Bi}[3]\right.$, $\left.\mathrm{Cd}_{3} \mathrm{As}_{2}[4,5]\right)$ and Weyl[6, 7] Semimetals. Due to existence of massless fermions in the dispersion relation, these materials provide a perfect opportunity for detailed study of quantum field theory phenomena, which were previously attributed to high-energy physics.

In this report we study the properties of Dirac Semimetals. Low energy fermionic degrees of freedom in this material are described by $N_{f}=2$ massless Dirac fermions with the dispersal relation

$$
E^{2}=v_{\|}^{2}\left(k_{x}^{2}+k_{y}^{2}\right)+v_{\perp}^{2} k_{z}^{2},
$$

${ }^{\star}$ Speaker, e-mail: kotov@itep.ru 
where $v_{\|}, v_{\perp}$ are Fermi velocities in the $(x, y)$ plane and $z$ direction correspondingly.

Due to the smallness of Fermi velocity $v_{F}$, electromagnetic interaction between quasiparticles has a large effective coupling constant $\alpha=\alpha_{e m} / v_{F} \sim 1$, thus it can lead to significant modification of the properties of Dirac semimetals. For instance, one can estimate $\alpha_{e f f} \sim 7$ for $\mathrm{Na}_{3} \mathrm{Bi}$ and $\alpha_{e f f} \sim 2$ for $\mathrm{Cd}_{d} \mathrm{As}_{2}$. So, this materials are strongly coupled and one should apply nonperturbative methods for studying the properties of these materials. This report is devoted to the lattice Quantum Monte Carlo simulations of Dirac Semimetals. We concentrate on two problems: the phase diagram of Dirac Semimetals in the plane effective coupling constant - Fermi velocity anisotropy and the conductivity of Dirac Semimetals in an external magnetic field. This report is the summary of the papers [8-10].

\section{Effective field theory}

Low-energy fermionic excitations of Dirac Semimetals can be described as $N_{f}=2$ massless 3+1D Dirac fermions with the dispersion relation (1). It is important to note the following features of the dispersion relation: smallness of the Fermi velocity $v_{F} \ll 1$ and it anisotropy $v_{\|} \neq v_{\perp}$. Due to the smallness of the Fermi velocity $v_{F} \ll 1$ one can neglect magnetic interaction and retardation effects. Thus, the interaction of quasiparticles in Dirac Semimetals is instantaneous Coulomb.

Based on the discussed above properties one can build low energy effective field theory for fermionic excitations in Dirac Semimetals. This effective field theory is described by the path integral $Z=\int D \bar{\psi} D \psi D A_{4} \exp \left(-S_{E}\right)$. The Euclidean action $S_{E}=S_{g}+S_{f}$ is written as the sum of gauge part $S_{g}$ and fermionic part $S_{f}$. As was explained above, the contribution of the spatial component $A_{i}$ is suppressed due to the smallness of the Fermi velocity $v_{F}$. For that reason the action $S_{E}$ includes only time-like component $A_{4}$. It is also convenient to rescale the time coordinate and timelike component of the electromagnetic field: $t \rightarrow t \cdot v_{F}, A_{4} \rightarrow A_{4} / v_{F}$. Taking all this into account, one can easily write the action of the theory:

$$
\begin{array}{r}
S_{g}=\frac{1}{8 \pi \alpha_{e f f}} \int d^{3} x d t\left(\partial_{i} A_{4}\right)^{2}, \\
S_{f}=\sum_{a=1}^{N_{f}} \int d^{3} x d t \bar{\psi}_{a}\left(\gamma_{4}\left(\partial_{4}+i A_{4}\right)+\zeta_{i} \gamma_{i} \partial_{i}\right) \psi_{a}=\sum_{a=1}^{N_{f}} \int d^{3} x d t \bar{\psi}_{a} D_{a}\left(A_{4}\right) \psi_{a}
\end{array}
$$

Here $\zeta_{i}=\frac{v_{F i}}{v_{F}} \sim 1$ are factors that incorporate Fermi velocity anisotropy and $\alpha_{e f f}=\frac{\alpha}{v_{F}}$ is an effective coupling constant.

\section{Lattice field theory formulation}

In the simulations we use the following discretization of the acton (2). As was discussed in [9], it is important to formulate and study the lattice field theory on anisotropic lattices $a_{s} \neq a_{t}$. For the electromagnetic field we used noncompact action: $S_{g}=\frac{\tilde{\beta}}{2} \sum_{x, i}\left(\theta_{4}(x)-\theta_{4}(x+i)\right)^{2}$. Here $\theta_{4}$ corresponds to $a_{t} A_{4}$ and $\tilde{\beta}$ is given by the expression $\tilde{\beta}=\beta \frac{a_{s}}{a_{t}}=\frac{v_{\perp}}{4 \pi \alpha \xi}, \beta=\frac{1}{4 \pi \alpha_{e f f}}$. Fermionic action is given by the expression:

$S_{f}=\bar{\psi}_{x} D_{x, y} \psi_{y}=\sum_{x}\left(m \bar{\psi}_{x} \psi_{x}+\frac{1}{2} \eta_{4}(x)\left[\bar{\psi}_{x} e^{i \theta_{4}(x)} \psi_{x+\hat{4}}-\bar{\psi}_{x+4} e^{-i \theta_{4}(x)} \psi_{x}\right]+\frac{1}{2} \sum_{i=1}^{3} \xi_{i} \eta_{i}(x)\left[\bar{\psi}_{x} \psi_{x+\hat{\beta}}-\bar{\psi}_{x+\beta} \psi_{x}\right]\right)$, 

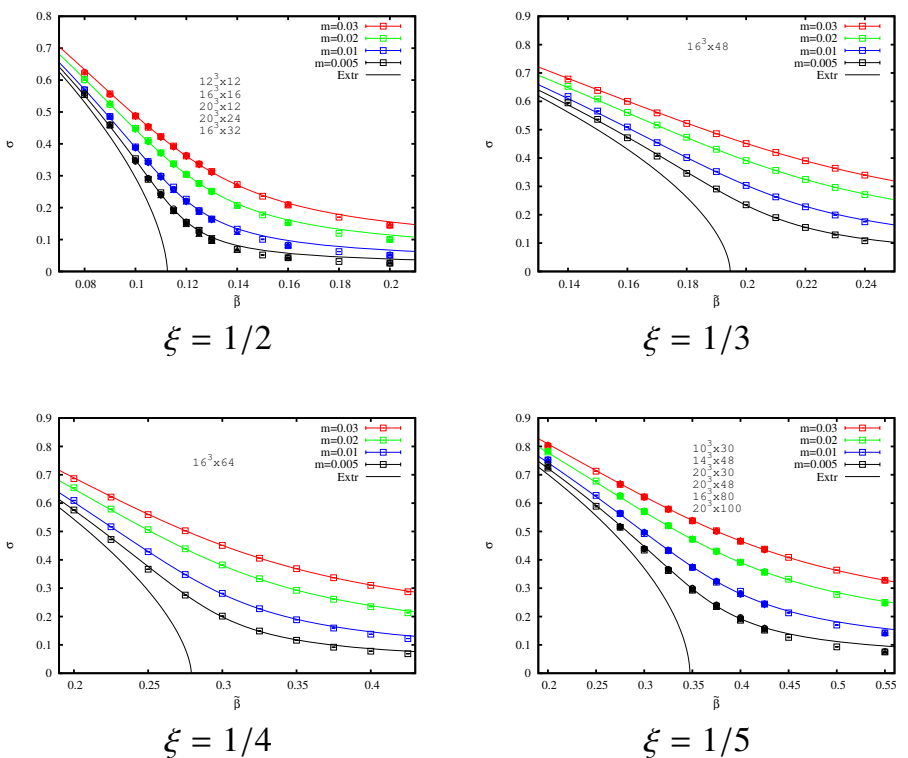

Figure 1. Dependence of the chiral condensate on the $\tilde{\beta}$ for various values of $m$. The points for different lattice sizes almost coincide. Errorbars are smaller than data points. Black line corresponds to extrapolation to $m=0$ obtained with the help of EoS (4). Different plots correspond to different values of $\xi=\frac{a_{t}}{a_{s}}$.

Here $\xi_{i}=\xi=a_{t} / a_{s}, i=1,2$ and in the third direction $\xi_{3}=\xi \frac{v_{\|}}{v_{\perp}}$. This action corresponds to $N_{f}=4$ fermion. For the reduction $N_{f}=4 \rightarrow 2$ we performed the standard rooting procedure. For numerical simulations the standard Hybrid Monte Carlo methods were applied[11].

\section{Phase diagram of Dirac Semimetals}

It is known that large values of effective coupling constant in Dirac semimetals $\alpha_{\text {eff }} \sim 1$ can cause dynamical generation of the fermion mass gap thus leading to the transition from semimetal to the insulator phase. In this section we are going to apply lattice field theory discussed in Ch.3 to study this phase transition and corresponding phase diagram of Dirac Semimetals. The order parameter for this phase transition is the chiral condensate $\sigma$, which is measured directly in our simulations. In the semimetal phase $\sigma \rightarrow 0$ in the chiral limit $m \rightarrow 0$, while in the insulator phase one expects nonzero value $\sigma(m \rightarrow 0) \neq 0$. To check finite volume effects we performed simulations at various lattice sizes $L_{s}^{3} \times L_{t}$. It was found, that within errorbars the measured values of the chiral condensate $\sigma$ coincide for all studied lattice sizes.

\subsection{Isotropic Fermi velocity}

First we study the case of isotropic Fermi velocity $v_{\|}=v_{\perp}$. It corresponds to $\xi_{1}=\xi_{2}=\xi_{3}=\xi$. To study the limit of zero temporal step $a_{t} \rightarrow 0$ we studied the dependence of the chiral condensate on the effective coupling constant for various values of $\xi=1 / 2,1 / 3,1 / 4,1 / 5$. To control finite volume effects simulations for two values of $\xi=1 / 2$ and $\xi=1 / 5$ were performed at various lattice sizes. We have found that for lattice sizes used in our simulations the measured values of the chiral condensate coincide with each other within errorbars, what allows us to state that finite volume effects are small. 

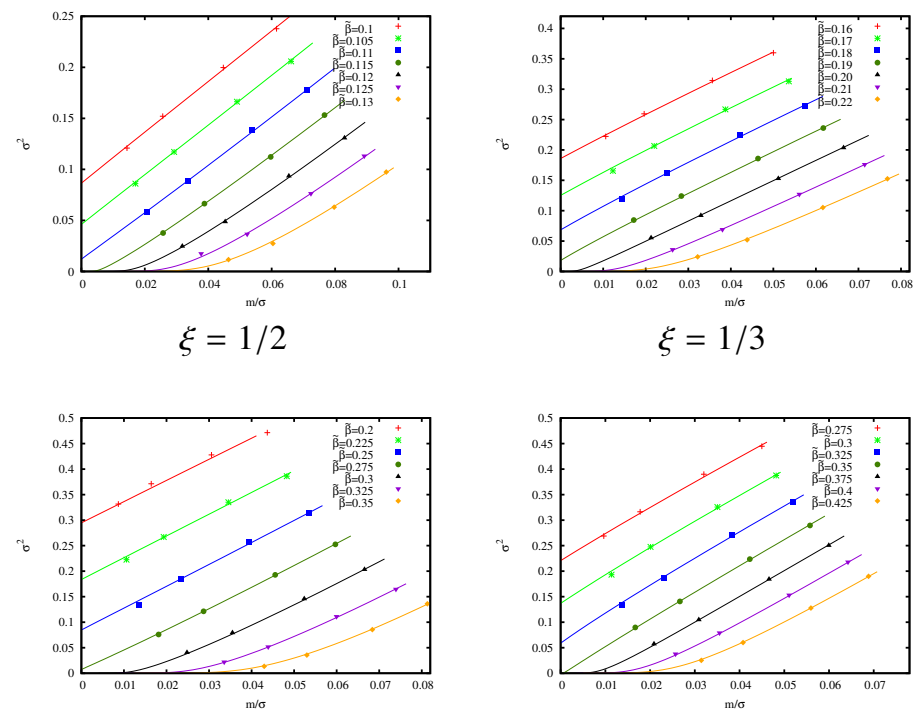

$\xi=1 / 4$

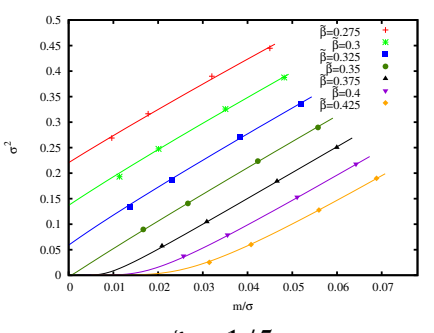

$\xi=1 / 5$

Figure 2. Fisher plot for the $\sigma^{2}$ with respect to the ratio $m / \sigma$. The lines correspond to the fit by EoS (4). Different plots correspond to different values of $\xi=\frac{a_{t}}{a_{s}}$.

The chiral condensate as a function of $\tilde{\beta}$ for various values of mass $m$ is presented in the Fig.1. All plots show the same behaviour and the formation of the chiral condensate in the chiral limit. To make some quantitative predictions we used the Equation of State(EoS), which parameterizes the dependence of the chiral condensate on mass and effective coupling constant in the vicinity of the phase transition (this EoS was successfully applied to strongly coupled QED[12] and graphene[13]):

$$
m X(\tilde{\beta})=Y(\tilde{\beta}) f_{1}(\sigma)+f_{3}(\sigma)
$$

Here $X(\tilde{\beta})=X_{0}+X_{1}\left(1-\tilde{\beta} / \tilde{\beta}_{c}\right), Y(\tilde{\beta})=Y_{1}\left(1-\tilde{\beta} / \tilde{\beta}_{c}\right)$ are expanded in the vicinity of critical $\tilde{\beta}_{c}$. For the functions $f_{1}$ and $f_{3}$ we allowed nonclassical critical exponents: $f_{1}(\sigma)=\sigma^{b}, f_{3}(\sigma)=\sigma^{\delta}$. Classical values of these exponents are $b=1$ and $\delta=3$. If one plots $\sigma^{2}$ vs $m / \sigma$ (Fisher plot) for various $\beta$, than the classical exponents would correspond to straight lines. The line that crosses the origin gives the critical value $\beta$. The Fisher plots for the considered values of $\xi$ are presented in Fig. 2. The lines on the plots correspond to the fit by EoS (4). Small deviations from straight lines can be seen, what means that the exponents should be close to classical. With the help of the EoS we determined the critical value $\beta_{c}$ as a function of $\xi$ (Fig. 3). The limit $\xi \rightarrow 0$ should be taken. Critical values of $\beta$ for two smallest values $\xi=1 / 4$ and $\xi=1 / 5$ are almost the same. As the final estimate we take half of the sum of $\beta_{c}$ obtained at $\xi=1 / 4$ (lattice size $16^{3} \times 64$ ) and $\xi=1 / 5$ (lattice size $16^{3} \times 80$ ). The obtained critical value is $\beta_{c}=0.06964(13)$, which corresponds to the critical coupling $\alpha_{c}=1.1414(21)$.

\subsection{Anisotropic Fermi velocity}

Discovered Dirac Semimetals $\mathrm{Na}_{3} \mathrm{Bi}$ and $\mathrm{Cd}_{3} \mathrm{As}_{2}$ possess anisotropy in Fermi velocity $v_{\|} \neq v_{\perp}$. Thus it is interesting how critical couping constant depends on the anistropy of the Fermi velocity. 


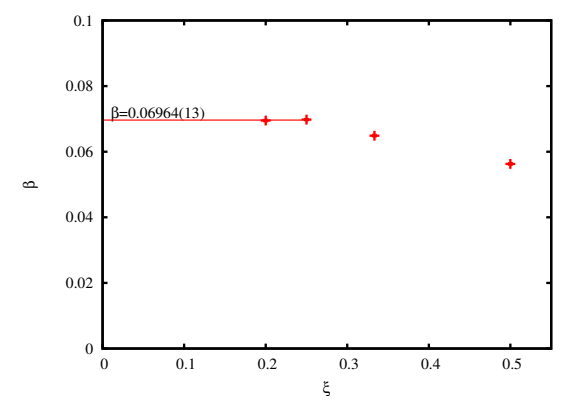

Figure 3. Critical $\beta_{c}=\tilde{\beta}_{c} \xi=\frac{1}{4 \pi \alpha_{e f f}}$ as a function of $\xi=\frac{a_{t}}{a_{s}}$. Correct effective theory is restored in the limit $\xi \rightarrow 0$. Red line corresponds to the average value for two points with the smallest $\xi=\frac{1}{4}$ and $\xi=\frac{1}{5}$.

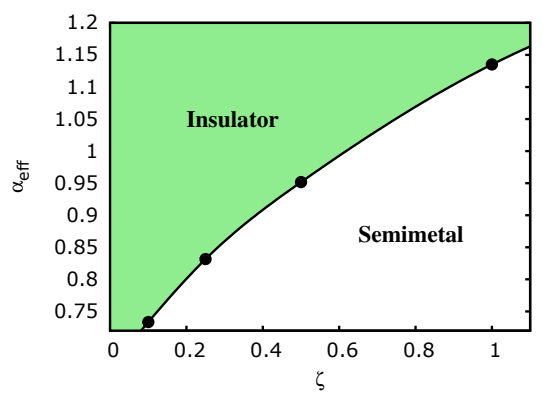

Figure 4. Dependence of the critical effective coupling $\alpha_{e f f}^{c}$ on Fermi velocity anisotropy $\zeta=\frac{v_{\|}}{v_{\perp}}$. Points correspond to the data obtained in the lattice simulations. Lines are to guide the eyes.

Study of the case with isotropic Fermi velocity has shown that the ratio $\xi=1 / 5$ is close to the limit $a_{t} \rightarrow 0$. For this reason we fix $\xi_{1}=\xi_{2}=\xi=1 / 5$ for two directions and vary $\xi_{3}=\zeta \xi, \zeta \in(0,1)$. It corresponds to the relation for Fermi velocity $v_{\|}=\zeta v_{\perp}$.

The dependence of the chiral condensate on $\beta$ is qualitatively similar to the isotropic case. Using the same procedure as in Ch.4.1 we determined the critical value of the coupling constant for these values of $\zeta$. This allowed us to draw the dependence of the critical coupling constant $\alpha$ on the value of the Fermi velocity anisotropy $\zeta$ (see Fig.4). Below the black line the system is in the semimetal phase, above this line the system is an insulator. It is also important to note, that the critical coupling constant $\alpha_{\text {eff }}$ decreases when anisotropy is increased $\zeta: 1 \rightarrow 0$. This can be understood from the following argument. When $\zeta$ goes to 0 the system becomes effectively two-dimensional. From quantum mechanics one may expect that the critical coupling for the $2 \mathrm{D}$ system is smaller than that for the $3 \mathrm{D}$ system, what is in agreement with our results.

\section{Chiral Magnetic Effect in Dirac Semimetals}

Discovery of Dirac Semimetals opens the possibility to study relativistic quantum field theory phenomena in condensed matter physics. Existence of Dirac points in dispersion relation allows to observe different manifestations of the quantum anomalies, and, in particular, the Chiral Magnetic $\operatorname{Effect}(\mathrm{CME})$. 

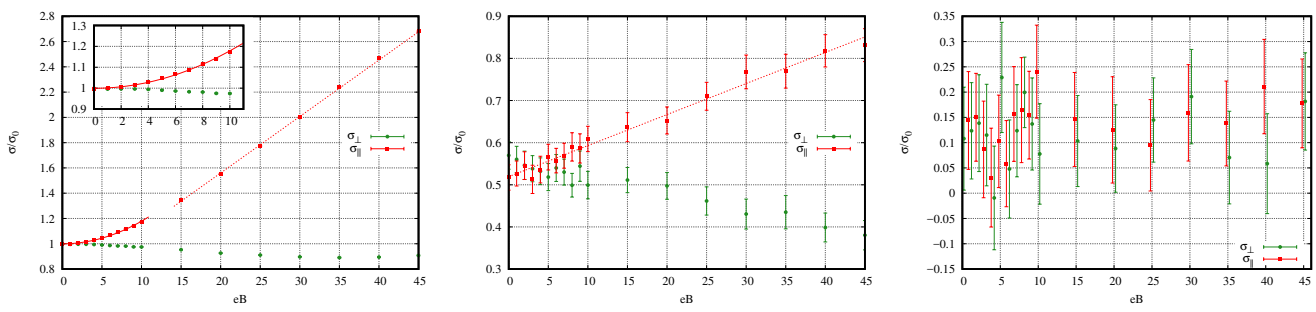

Figure 5. The ratios $\sigma_{\|, \perp} / \sigma_{0}$ as a function of the external magnetic field $e B$ for three values of $\alpha_{\text {eff }}=0.32$ (semimetal phase), $\alpha_{e f f}=1.27$ (the onset of the insulator phase), $\alpha_{e f f}=2.55$ (insulator phase). The $\sigma_{0}$ is the conductivity at $\alpha_{e f f}=0.32$ at zero magnetic field. The external magnetic field $e B$ is shown in units $\frac{2 \pi}{\left(a_{s} L_{s}\right)^{2}}$. Red points correspond to conductivity parallel to the magnetic field $\sigma_{\|}$, green points give conductivity in the perpendicular direction $\sigma_{\perp}$. Red lines correspond to quadratic and linear fit.

To observe the CME external parallel electric $\mathbf{E}$ and magnetic $\mathbf{B}$ fields are turned on. Due to the chiral anomaly this leads to the chiral density[14] $\rho_{5}=\frac{e^{2}}{4 \pi^{2} \hbar^{2} c} \mathbf{E} \cdot \mathbf{B} \tau . \tau$ is the chirality relaxation time[15, 16]. In the limit of small electric field $\mathbf{E}, \rho_{5}$ is also small and one can write the Equation of State (EoS) as $\rho_{5}=\mu_{5} \chi(T, B)+O\left(\mu_{5}^{3}\right)$, where $\chi(T, B)$ is the function of temperature and magnetic fields. It is reasonable to expect that in the limit of small magnetic field $e B / T^{2} \rightarrow 0, \chi$ is described by $\chi \sim T^{2}$. In the opposite limit of large $e B / T^{2} \rightarrow \infty, \chi$ is determined by the degeneracy on the lowest Landau level and one can expect that $\chi \sim e B$. Within external magnetic field, nonzero chiral density, parameterized by the chiral chemical potential leads to the electric CME current $\mathbf{j}=\frac{e^{2}}{2 \pi^{2}} \mu_{5} \mathbf{B}$. This implies additional term in the conductvity if $\mathbf{E}$ is parallel to $\mathbf{B}$ :

$$
\sigma^{z z}=\sigma_{O}+\frac{e^{4}}{8 \pi^{4} \hbar^{2} c} \frac{\tau}{\chi(T, B)} B^{2} .
$$

At the same time, in the perpendicular direction additional term $\sigma_{C M E}^{x x}=0$ is zero.

This growth of conductivity with the magnetic field is a clear consequence of the CME, which can not be described classically. Due to the discussed behaviour of $\chi$ in the limit of large and small magnetic fields, one can see that for small magnetic field $\sigma^{z z}$ rises quadratically with $B$. For large magnetic fields this behaviour changes to linear growth. Experimental observation of the CME in Dirac Semimetals via the measurements of conductivity was reported in $[14,17,18]$.

Interaction between quasiparticles in Dirac semimetals is strong, what can lead to significant modification of the above formulae. Moreover, as was discussed earlier in the Chapter 4, at large enough coupling constants the system becomes an insulator due to the chiral symmetry breaking. In this respect it is interesting to study the CME for various coupling constants and in various phases.

To measure the electric conductivity within lattice simulations we measured the Euclidean correlator $C_{i}(t)$ of the spatial components of electric current $j_{i}=\bar{\psi} \gamma_{i} \psi, i=1,2,3$ (note that in this formula there is no summation over index $i$ ):

$$
C_{i}(t)=\int d^{3} \bar{x}\left\langle j_{i}(\bar{x}, t) j_{i}(0)\right\rangle
$$

The correlator (6) is related to the conductivity $\sigma_{i i}(\omega)$ by means of Kubo relation[19]: $C_{i}(t)=$ $\int_{0}^{\infty} \frac{d \omega}{2 \pi} K(\omega, t) \sigma_{i i}(\omega)$, where $K(\omega, t)=\frac{2 \omega \cosh \left(w\left(\frac{1}{2 T}-t\right)\right)}{\sinh \left(\frac{\omega}{2 T}\right)}$ is the standard thermal kernel. In order to study the CME one needs the conductivity $\sigma_{i i}(\omega)$ at small frequencies. In this paper we are going to use 
midpoint calculation of the conductivity[19]. Within this approach one estimates the $\sigma_{i i}(\omega)$ at small frequencies as weighted average of the conductivity which is related to the correlation function at the midpoint. Physically the estimator $\bar{\sigma}_{i i}$ averages the conductivity over the interval $\sim$ few $\times T$ :

$$
\bar{\sigma}_{i i}=\int_{0}^{\infty} \frac{d \omega}{2 \pi} \frac{2 \omega}{\sinh \frac{\omega}{2 T}} \sigma_{i i}(\omega) / \int_{0}^{\infty} \frac{d \omega}{2 \pi} \frac{2 \omega}{\sinh \frac{\omega}{2 T}}=\frac{1}{\pi T^{2}} C_{i}\left(\frac{1}{2 T}\right)
$$

Here we study only the isotropic Fermi velocity. In the calculations we use the lattice size $L_{s}=$ $L_{t}=20$, the asymmetry $\xi=a_{t} / a_{s}=1 / 4$ (one can expect that this value is close to the limit $\xi \rightarrow 0$ ) and the fermion mass in lattice units $m=0.0025$. The following effective coupling constants were studied: $\alpha_{e f f}=0.32$ (semimetal phase), 1.27 (insulator but close to the transition point), 2.55 (insulator phase).

In Fig. 5 we plot the ratios $\sigma_{\|, \perp} / \sigma_{0}$ vs the magnetic field $e B$ for various values of the $\alpha_{e f f}$. The $\sigma_{0}$ is conductivity $\sigma_{x x}$ at $\alpha_{e f f}=0.32$ at zero magnetic field. Red points on these figures correspond to conductivity parallel to the magnetic field $\sigma_{\|}$, green points give conductivity in the perpendicular direction $\sigma_{\perp}$. For $\alpha_{e f f}=0.32$ (semimetal phase) it is clearly seen, that $\sigma_{\|}$grows whereas $\sigma_{\perp}$ drops with the magnetic field. So we see positive magnetoresistance for the $\sigma_{\perp}$ and negative magnetoresistance for the $\sigma_{\|}$what agrees with our expectation and the experiment [14]. Notice that there are two regimes of the dependence of the conductivity $\sigma_{\|}(B)$ on the magnetic field. For small magnetic field the $\sigma_{\|}(B)$ is quadratically rising function, while for larger magnetic field it is linear. This behaviour of the $\sigma_{\|}$is in agreement with our expectation from formula (5). This brings us to the conclusion that we observe the CME in the lattice simulation of Dirac semimetals in the semimetal phase.

From Fig. 5 it is seen that for $\alpha_{e f f}=1.27$ (the onset of the insulator phase) the $\sigma_{\|}(B)$ and $\sigma_{\perp}(B)$ behave similarly to the semimetal phase, but their absolute values are smaller and the CME is weaker. Finally for the insulator phase $\left(\alpha_{e f f}=2.55\right)$ it is seen that the conductivity is close to zero, it does not depend on the magnetic field and we do not observe the CME.

\section{Conclusions}

Effective field theory of Dirac Semimetals is formulated. Lattice discretized version of this theory is constructed and it is shown that in the limit of zero temporal lattice spacing $a_{t} \rightarrow 0$ it reproduces low energy effective theory with correct number of degrees of freedom.

The phase diagram of Dirac Semimetals in the plane effective coupling constant - Fermi velocity anisotropy is studied by means of supercomputer simulations. Systematic uncertainties due to finite volume effects and nonzero temporal lattice spacing are under control. The value of the critical coupling constant for semimetal-insulator phase transition and its dependence on the Fermi velocity anisotropy is determined. Tentative phase diagram of Dirac Semimetals is drawn.

The second part is aimed at the numerical study of Chiral Magnetic Effect in Dirac Semimetals. Its manifestation in Dirac Semimetals is large (magneto-)conductivity in the direction parallel to the magnetic field. Conductivity of Dirac Semimetals in external magnetic field is measured. Calculations were carried out in three regimes: in semimetal phase, in insulator phase and at semimetal insulator transition. In semimetal phase the $\sigma_{\|}$grows whereas $\sigma_{\perp}$ drops with magnetic field. Similar behaviour was observed in the onset of the insulator phase but the dependence is weaker and the conductivity is smaller. Finally in the insulator phase the conductivities $\sigma_{\|, \perp}$ are close to zero and do not depend on magnetic field. So, we observe manifestation of CME current in semimetal phase, weaker manifestation of CME in the onset of insulator phase. We do not observe CME in the insulator phase.

Acknowdedgements. The work supported by the RSF grant under contract 16-12-10059. Numerical simulations were carried out on GPU cluster of NRC Kurchatov Institute and at MSU supercomputer "Lomonosov". 


\section{References}

[1] K.S. Novoselov, A.K. Geim, S.V. Morozov, D. Jiang, Y. Zhang, S.V. Dubonos, I.V. Grigorieva, A.A. Firsov, Science 306, 666 (2004), http://science.sciencemag.org/content/306/5696/666.full.pdf

[2] A.K. Geim, K.S. Novoselov, Nat Mater 6, 183 (2007)

[3] Z.K. Liu, B. Zhou, Y. Zhang, Z.J. Wang, H.M. Weng, D. Prabhakaran, S.K. Mo, Z.X. Shen, Z. Fang, X. Dai et al., Science 343, 864 (2014), http://science.sciencemag.org/content/343/6173/864.full.pdf

[4] M. Neupane, S.Y. Xu, R. Sankar, N. Alidoust, G. Bian, C. Liu, I. Belopolski, T.R. Chang, H.T. Jeng, H. Lin et al., Nat Commun 5 (2014)

[5] S. Borisenko, Q. Gibson, D. Evtushinsky, V. Zabolotnyy, B. Büchner, R.J. Cava, Phys. Rev. Lett. 113, 027603 (2014)

[6] S.Y. Xu, I. Belopolski, N. Alidoust, M. Neupane, G. Bian, C. Zhang, R. Sankar, G. Chang, Z. Yuan, C.C. Lee et al., Science 349, 613 (2015), http://science.sciencemag.org/content/349/6248/613.full.pdf

[7] S.Y. Xu, I. Belopolski, D.S. Sanchez, C. Zhang, G. Chang, C. Guo, G. Bian, Z. Yuan, H. Lu, T.R. Chang et al., Science Advances 1 (2015), http://advances.sciencemag.org/content/1/10/e1501092 . full.pdf

[8] V.V. Braguta, M.I. Katsnelson, A.Yu. Kotov, A.A. Nikolaev, Phys. Rev. B94, 205147 (2016), 1608.07162

[9] V.V. Braguta, M.I. Katsnelson, A.Yu. Kotov (2017), 1704.07132

[10] D.L. Boyda, V.V. Braguta, M.I. Katsnelson, A.Yu. Kotov (2017), 1707.09810

[11] I. Montvay, G. Munster, Quantum fields on a lattice (Cambridge University Press, 1997), ISBN 9780521599177, 9780511879197, http://www.cambridge.org/uk/catalogue/ catalogue . asp?isbn=0521404320

[12] M. Gockeler, R. Horsley, V. Linke, P.E.L. Rakow, G. Schierholz, H. Stuben, Nucl. Phys. B487, 313 (1997), hep-lat/9605035

[13] J.E. Drut, T.A. Lahde, Phys. Rev. Lett. 102, 026802 (2009), 0807.0834

[14] Q. Li et al., Nature Phys. 12, 550 (2016), 1412.6543

[15] C. Manuel, J.M. Torres-Rincon, Phys. Rev. D92, 074018 (2015), 1501.07608

[16] M. Ruggieri, G.X. Peng, M. Chernodub, Phys. Rev. D94, 054011 (2016), 1606.03287

[17] C.Z. Li et al., Nat. Commun. 6, 10137 EP (2015)

[18] H. Li et al., Nat. Commun. 7, 10301 EP (2016)

[19] P.V. Buividovich, M.I. Polikarpov, Phys. Rev. B86, 245117 (2012), 1206.0619 